\title{
(2) OPEN ACCESS \\ Direct selective laser trabeculoplasty in open angle glaucoma study design: a multicentre, randomised, controlled, investigator-masked trial (GLAUrious)
}

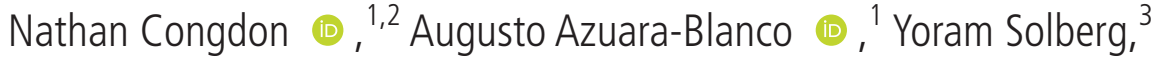 \\ Carlo E Traverso, ${ }^{4}$ Michele lester (1) , ${ }^{4}$ Carlo Alberto Cutolo (1) , ${ }^{4}$ Alessandro Bagnis, ${ }^{4}$ \\ Tin Aung, ${ }_{1}^{5}$ Scott J Fudemberg, ${ }^{6}$ Richard Lindstrom, ${ }^{7,8}$ Thomas Samuelson, ${ }^{7}$ \\ Kuldev Singh, ${ }^{9}$ Eytan Z Blumenthal, ${ }^{10,11}$ Gus Gazzard, ${ }^{12,13}$ On behalf of GLAUrious \\ study group
}

For numbered affiliations see end of article.

\section{Correspondence to}

Dr Nathan Congdon, Centre for Public Health, Queen's University Belfast, Belfast, Guangdong, UK; ncongdon1@gmail.com

Received 5 April 2021 Accepted 10 July 2021
D) Check for updates

(C) Author(s) (or their employer(s)) 2021. Re-use permitted under CC BY-NC. No commercial re-use. See rights and permissions. Published by BMJ.

To cite: Congdon N, AzuaraBlanco A, Solberg Y, et al. Br J Ophthalmo/ Epub ahead of print: [please include Day Month Year]. doi:10.1136/ bjophthalmol-2021-319379

\begin{abstract}
Introduction Laser trabeculoplasty is an effective and widely used treatment for glaucoma. A new laser technology, the Eagle direct selective laser trabeculoplasty (DSLT) device, may provide automated, fast, simple, safe and effective laser treatment for glaucoma in a broader range of clinical settings. This trial aims to test the hypothesis that translimbal DSLT is effective and not inferior to selective laser trabeculoplasty (SLT) in reducing intraocular pressure $(\mathrm{IOP})$ in open angle glaucoma (OAG).
\end{abstract}

Methods and analysis This is a multicentre, randomised, controlled, investigator-masked study. The primary efficacy outcome is intergroup difference in mean change from baseline IOP measured at 6 months. Secondary outcomes include mean percentage reduction in IOP at 3, 6 and 12 months; proportion of participants with at least $20 \%$ reduction in IOP from baseline at 6 months; change in ocular hypotensive medications at 12 months and evaluation of safety. Participants were aged $>=40$ years with $O A G$, including exfoliative or pigmentary glaucoma, or ocular hypertension with untreated or washed out IOP $22-35 \mathrm{~mm} \mathrm{Hg}$. Treatments: DSLT: 120 shots, 3 ns, $400 \mu \mathrm{m}$ spot size, energy 1.4-1.8 $\mathrm{mJ}$ delivered at the limbus over $2 \mathrm{~s}$. SLT: approximately 100 shots, 3 ns, $400 \mu \mathrm{m}$ spot size administered 360 degrees at the limbus using any gonioscopy lens, energy 0.3-2.6 mJ. A sample size of 164 is sufficient to detect a non-inferiority margin of $1.95 \mathrm{~mm} \mathrm{Hg}$ for change from baseline IOP.

Clinical trial registration number NCT03750201, ISRCTN14033075.

\section{INTRODUCTION}

Glaucoma is a chronic, initially asymptomatic disease that may result in progressive optic nerve damage. An estimated 76 million people suffer from glaucoma worldwide, ${ }^{1}$ making it the leading cause of irreversible blindness globally. ${ }^{2}$ Although the damage is irreversible, further deterioration ('progression') may be prevented or delayed by reducing intraocular pressure (IOP). The goal of glaucoma treatment is to maintain the patient's visual function with minimal impact on quality of life, and ideally, in the most cost-effective fashion.
Treatment of glaucoma with topical hypotensive medications depends on patient adherence and may be associated with side effects, inconvenience and financial burden to the patient. As a result, up to $50 \%-80 \%$ of patients fail to adhere to treatment over a 12-month period. ${ }^{3}$ This study is addressing the top research priority of the James Lind Alliance: 'What are the most effective treatments for glaucoma and how can treatment be improved?'.

Treatment of glaucoma with laser trabeculoplasty (LTP), such as selective laser trabeculoplasty (SLT), has been available for many years. Treatment with SLT compared with medical treatment offers costeffective drop-free disease control for $74 \%$ of patients at 3 years when used as an initial therapy, as reported in a large randomised controlled trial. ${ }^{5}$ The IOP reduction effect is still present in $50 \%$ of patients at 5 years, and treatment is repeatable. ${ }^{6}$ SLT targets the trabecular meshwork (TM) and is designed to reduce IOP by improving aqueous outflow. The procedure lasts approximately 5-10 min and consists of 50-100 separate laser 'spots' delivered through a manually rotated mirrored lens (goniolens) placed on the cornea. Although SLT is considered to be a low-risk procedure, the goniolens requires use of a coupling gel and prolonged contact with the patient's eye that can result in epithelial damage, blurred vision and discomfort, and carries a small but significant infection risk. Although the effectiveness of SLT as an initial treatment for primary open angle glaucoma (POAG) and ocular hypertension $(\mathrm{OHT})$ has been demonstrated in clinical trials, ${ }^{5} 7$ the procedure requires expertise which limits its widespread use.

Recently, Geffen et $_{\text {al }}^{8}$ investigated the safety and efficacy of performing translimbal SLT without the use of a goniolens. In this small study, they were able to show that this technique appeared to be as efficacious as conventional SLT, however, taking less time and possibly causing fewer complications, possibly due to its non-contact methodology and avoidance of transcorneal laser path.

The current protocol uses a new device, the External Automatic Glaucoma Laser (Eagle): a translimbal, direct selective laser trabeculoplasty (DSLT) system. Non-contact treatment automatically detects and directs laser energy through the 
limbus overlying the TM. The entire 360 degrees of the TM is treated simultaneously in about $2 \mathrm{~s}$.

\section{METHODS}

This is a multicentre, randomised, controlled, investigatormasked trial designed to evaluate the relative safety and efficacy of translimbal DSLT compared with conventional SLT for glaucoma.

\section{Study outcomes}

The primary efficacy outcome is the difference between the two treatment groups between the mean (washed out for medicated patients) baseline IOP and the mean (washed out for medicated patients) IOP measured at 6 months. Secondary efficacy outcomes include between-group differences in mean percentage reduction in IOP at 3, 6 and 12 months; the proportion of participants with at least $20 \%$ reduction in IOP from baseline at 6 months; and the change in mean number of topical hypotensive medications at 12 months compared with baseline. All treatments will be administered by an unmasked ophthalmologist. All post-randomisation IOP measures will either be collected using a masked technique or by a masked ophthalmologist.

Safety measures include all ocular adverse events (AEs). Quality of life and patient satisfaction will be evaluated with the Glaucoma Quality of Life (GQL-9) ${ }^{910}$ and a standardised questionnaire.

\section{Ethics and regulatory approvals}

The trial will be conducted according to the World Medical Association Declaration of Helsinki and is in receipt of full regulatory authority and research ethics committee (REC) approval in each country and site. The protocol is prospectively registered on a publicly accessible database (Protocol \# 2017_01, Clintrials.gov \# NCT03750201, ISRCTN14033075). Potential participants will be provided with the REC-approved patient information sheet, and given sufficient time to review and ask questions before providing written informed consent ahead of any study procedures.

\section{Study population}

The study population comprises participants aged over 40 years with OAG, including exfoliative or pigmentary glaucoma, or $\mathrm{OHT}$ who are able to give informed consent to participate for 12 months. IOP at randomisation must fall between 22 and 35 $\mathrm{mm} \mathrm{Hg}$ inclusive in either an untreated or washed-out state. Participants must have gonioscopically visible scleral spur for 360 degrees and clear visualisation of the perilimbal sclera for 360 degrees with a vertical cup to disc ratio of less than 0.8 . Patients with contraindications to SLT are excluded as are patients with angle closure glaucoma, congenital or developmental glaucoma, secondary glaucoma (except exfoliative or pigmentary glaucoma) or those with the presence of any peripheral anterior synechiae in the study eye. Patients must be able to undertake a reliable visual field test (defined as fixation losses, false positives and false negatives less than 33\%). Any of the following visual field findings using the Humphrey visual field (HVF) analyser and Swedish Interactive Thresholding Algorithm (SITA) standard 24-2 programme would exclude a patient: a visual field mean deviation of less than $-12 \mathrm{~dB}$, greater than $75 \%$ of points depressed below the $5 \%$ level and greater than or equal to $50 \%$ of points depressed below the $1 \%$ level on the pattern deviation plot, at least $50 \%$ of points, that is, 2 or more, within the central 5 degrees with a sensitivity of $0 \mathrm{~dB}$ on the decibel plot and points within the central 5 degrees of fixation with a sensitivity greater than $15 \mathrm{~dB}$ in both hemifields on the decibel plot. The mean defect in the fellow eye must be greater than $-12 \mathrm{~dB}$. Participants must not have any other clinically significant disease in either eye, prior incisional glaucoma surgery in the study eye, any significant cataract or cataract surgery in the last 6 months, prior corneal refractive surgery, prior SLT, significant amblyopia or dense pigmentation or haemorrhage in the perilimbal conjunctiva or anterior sclera. Other exclusions will include pregnancy, participation in another clinical trial other than a substudy of this trial, clinically relevant systemic disease or the anticipated need for other ocular surgery.

\section{Device description}

DSLT is a treatment modality for POAG, which uses similar laser parameters as SLT and has similar treatment indications. As in SLT, DSLT employs frequency-doubled, Q-switched Nd:YAG laser with a wavelength of $532 \mathrm{~nm}$. During the procedure, laser energy is delivered to the TM. This increases the permeability of the TM endothelial cells and thereby increases outflow, resulting in reduced IOP. In contrast to SLT, the DSLT treatment uses the Eagle system to direct the laser energy directly through the limbus to the TM without the need for a delivery device (gonioscopy lens) or any contact with the participant's eye. Laser energy is administered through a full 360 degrees, almost completely automatically by the Eagle system, with only the operator's rough alignment of the eye and approval of the device's automatically acquired target. Laser treatment lasts for about $2 \mathrm{~s}$ with 120 laser pulses of preset $3 \mathrm{~ns}$ and a preset $400 \mu \mathrm{m}$ spot size will be used, with the laser energy of $1.4-1.8 \mathrm{~mJ}$ delivered to the limbus. For the SLT, approximately 100 shots of a preset 3 ns duration and a preset $400 \mu \mathrm{m}$ spot size will be used 360 degrees around the limbus, with the laser energy varied from 0.3 to 2.6 $\mathrm{mJ}$ by the clinician using any laser gonioscopy lens.

The trial has started using the prototype device, which was used in the first human study. ${ }^{9}$ During this trial, the Eagle system became available. The Eagle system has the same functionality as the prototype, but with increased usability, namely, the device is now a smaller and lighter table top unit.

\section{Enrolment, randomisation and data acquisition}

Randomisation will be per site, with lists generated as a 1:1 random algorithm by the study statistician (two sequences according to whether or not the participant is receiving betablockers) and sealed envelopes provided to site. All treatments will be administered by unmasked ophthalmologists, although masked investigators will collect post randomisation IOP and study assessments. It is not possible to mask participants due to the varying nature of the two treatments. Figure 1 summarises the study design and patient flow.

After providing written informed consent, potential participants will be screened to ensure eligibility.

At this visit, the following examinations will be performed on both eyes:

- Goldmann applanation tonometry.

- Corrected visual acuity (CVA) using the ETDRS alphabet chart on a backlit light box.

- Slit-lamp examination of the anterior segment.

- Gonioscopy.

- Fundus examination.

- HVF examination using the 24-2 SITA standard programme.

- Recording of current ocular hypotensive medications and systemic medications (type and dosage).

If a participant is currently using topical ocular medications, IOP will be reassessed in a post-washout visit after a washout 


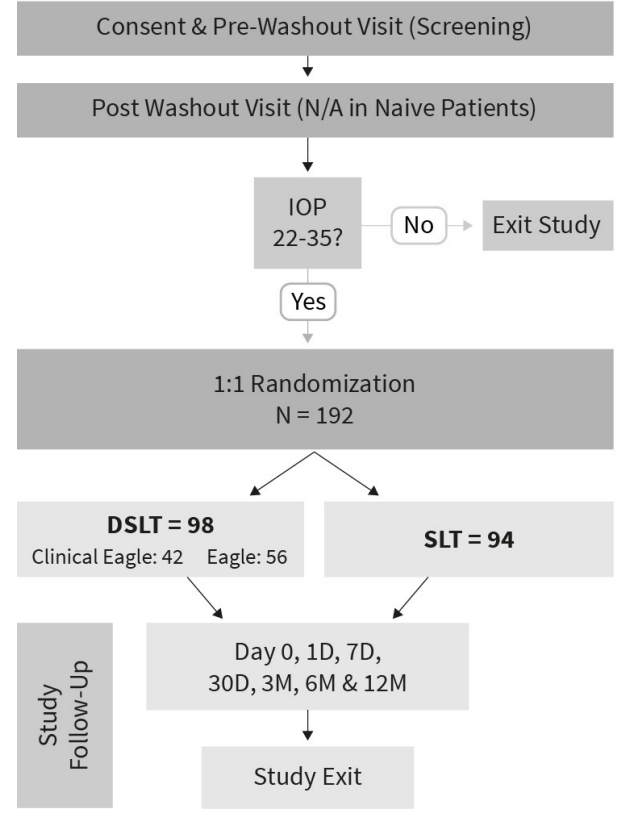

Figure 1 Flowchart depicting the enrolment and progression of participants through the trial. DSLT, direct selective laser trabeculoplasty; IOP, intraocular pressure; SLT, selective laser trabeculoplasty.

period of length determined by the type of medication(s) being used (up to 28 days).

During the washout period, additional visits can be conducted at the discretion of the investigator, to ensure continued safety, particularly for patients considered at risk for an unacceptably high IOP spike during washout.

If during one of these visits it is found that the participant's IOP increases to an unsafe level (at the investigators' discretion), ocular hypotensive medication will be reintroduced and they will be followed for safety until any AEs are resolved or stabilised, and then exited from the study.

Following washout, the eligibility of patients is confirmed and the threshold IOP value is determined by the investigators' clinical judgement. Participants will then be randomised 1:1 to SLT or DSLT. If both eyes are washed out and found to be eligible for inclusion, the principal investigator will enrol the eye with the highest post-washout IOP. If both eyes have the same IOP, the eye with the more advanced disease will be chosen. Standard glaucoma treatment will be (re)initiated in the non-study eye at the discretion of the investigator.

\section{Study interventions}

As a prophylaxis against laser-induced IOP elevation, apraclonidine or brimonidine drops will be administered (45-60 min and $10 \mathrm{~min}$ before treatment) as well as a drop of pilocarpine $(60 \mathrm{~min}$ before treatment).

Following treatment, the following assessments will be made on the study eye only:

- IOP immediately following treatment.

- IOP 1-2 hours after treatment (additional care at the discretion of the investigator).

- CVA using ETDRS.

- Slit-lamp examination with grading of anterior chamber cells $(0$ to +4$)$ and flare $(0$ to +4$)$.

Patients will be asked to provide feedback following treatment to assess satisfaction, pain and willingness to undergo the treatment again (graded using a Likert scale).
Following the treatment, participants will be instructed to use topical non-steroidal anti-inflammatory medications in the treated eye for 1 week at the discretion of the investigator.

Scheduled follow-up and assessment visits are planned at 1 day, 1 week, and 1, 3, 6 and 12 months following treatment. Patients receiving glaucoma medications will be asked to stop these 28 days before the 6-month visit.

Gonioscopic examination will be repeated at the 6-month follow-up visit to detect any changes to the anterior chamber angle after the laser treatment.

At each visit, the IOP will be assessed and compared with the threshold IOP limit value, if this value is reached, topical hypotensive medications will be initiated, starting with prostaglandin, then beta-blocker and other medications according to standard of care.

Patients will be asked to repeat the GQL-9 questionnaire at the 6-month visit and they will assess their willingness to repeat the treatment and whether they would recommend the treatment to a friend with glaucoma.

Treatment modification will be allowed for safety reasons if IOP becomes uncontrolled after treatment. There will be no reintroduction of medication for at least 1 month after treatment unless, in the opinion of the investigator, there is immediate risk to the health of the participant.

If cataract surgery, trabeculectomy or any other glaucoma surgery is required during the study follow-up period, the participant will undergo such treatment as deemed necessary (at the discretion of the investigator) and will be considered a treatment failure. She/he will continue to be followed up in the study glaucoma clinics thereafter.

Safety will be evaluated throughout the study consisting of recording of all AEs. An independent medical monitor will be responsible for overseeing safety and for evaluating all AEs reported to the sponsor. AEs and serious AEs are defined according to the International Conference on Harmonization E6 (R2) and International Organization for Standardization 14155:2011(E) definition.

\section{Statistical analyses and sample size calculation}

A total of 164 patients will be required to reject a non-inferiority null hypothesis that DSLT change-from-baseline IOP is more than $1.95 \mathrm{mmHg}$ worse (less) than the SLT change-frombaseline IOP. The primary hypothesis will be tested at 6 months post-treatment using the estimated difference in change-frombaseline IOP between study groups (DSLT and SLT) with $80 \%$ power and the associated 95\% two-sided CI, a fitted analysis of covariance (ANCOVA) model will adjust for baseline IOP and for the use of beta-blocker drops in the fellow eye.

The sample size calculation was based on a non-inferiority margin of $1.95 \mathrm{~mm} \mathrm{Hg}$ and an anticipated SD of 3.5. ${ }^{10}$ The noninferiority margin of $1.95 \mathrm{~mm} \mathrm{Hg}$ was chosen based on clinical judgement as IOP measurement differences of up to $2 \mathrm{~mm} \mathrm{Hg}$ are considered to be non-clinically significant. ${ }^{11} 12$ Other literature reports a predefined non-inferiority margin of $1-2.5 \mathrm{~mm} \mathrm{Hg} .{ }^{13}$

The mean percentage reduction in IOP from baseline will be summarised by treatment group at 3, 6 and 12 months after enrolment. The proportion of participants with at least $20 \%$ reduction in IOP from baseline will be summarised at 6 months with associated 95\% CI. The mean number of medications will be calculated for each group before treatment and at 12 months, and compared between groups. The GQL-9 questionnaire results will be summarised in appropriate tables by treatment group.

AEs will be coded and presented according to relatedness, severity and seriousness. Rates of AEs (on a per person basis) will be 
calculated, and 95\% CIs for the rates, and for the difference between treatment groups, will be produced using score-based methods.

\section{CONCLUSION}

Laser trabeculoplasty is an approved treatment available to patients with OAG that can be used with or without ocular hypotensive medication to reduce IOP. The safety and effectiveness of SLT has been well demonstrated in prior studies. ${ }^{13-16}$ Due to its IOP-lowering effect, SLT may result in a decrease or elimination of ocular hypotensive medications. SLT may cause inflammatory reactions, including cystoid macular oedema and, more commonly, anterior chamber inflammation. ${ }^{17-19}$ The inflammation is usually mild to moderate in its severity and transient, with reductions in intensity seen within 24 hours post-treatment, and resolution in most eyes within 5 days. ${ }^{20}$

Another risk associated with SLT is the occurrence of postoperative IOP elevation. This usually occurs in the immediate postoperative phase (1-2 hours) and careful patient selection and prophylactic treatment can greatly reduce their occurrence. ${ }^{21}$

DSLT is performed without physically touching the cornea, and preliminary studies have shown that it is a more rapid and simpler technique when compared with SLT. ${ }^{8}$ Further, it has been suggested that it could be more easily administered in patients in whom contact techniques prove problematic or where anatomical obstacles exist, such as narrow iridocorneal angles or prominent facial bones. ${ }^{8}$ The same study demonstrated similar IOP-lowering effect in both DSLT and SLT, and suggested that DSLT resulted in less corneal injury, inflammation and postoperative discomfort when compared with standard SLT.

Benefits of DSLT may include a faster and simpler treatment, less postoperative inflammation and less subjective discomfort. In addition, the procedure does not require use of a goniolens and therefore has the potential to increase the accessibility of glaucoma care throughout the world. This study will determine the efficacy and safety of the new Eagle system.

\author{
Author affiliations \\ ${ }^{1}$ Centre for Public Health, Queen's University Belfast, Belfast, UK \\ 'Zhongshan Opthalamic Center, Sun Yat-Sen University, Guangzhou, China \\ ${ }^{3}$ Belkin Vision, St. Yavne, Israel \\ ${ }^{4}$ Department of Neurosciences, Rehabilitation, Ophthalmology, Genetics, Maternal \\ and Child Health, University of Genoa, Genova, Italy \\ ${ }^{5}$ Singapore Eye Research Institute, Singapore National Eye Centre, Singapore \\ ${ }^{6}$ Wills Eye Hospital, Thomas Jefferson University, Philadelphia, Pennsylvania, USA \\ ${ }^{7}$ Minnesota Eye Consultants, Minneapolis, Minnesota, USA \\ ${ }^{8}$ Department of Ophthalmology and Visual Neurosciences, University of Minnesota, \\ Minneapolis, Minnesota, USA \\ ${ }^{9}$ Ophthalmology, Stanford University School of Medicine, Stanford, California, USA \\ ${ }^{10}$ Department of Ophthalmology, Rambam Health Care Campus, Haifa, Israel \\ ${ }^{11}$ Ruth and Bruce Rappaport Faculty of Medicine, Technion Israel Institute of \\ Technology, Haifa, Israel \\ ${ }^{12}$ NIHR Moorfields Biomedical Research Centre, and Moorfields Eye Hospital City \\ Road Campus, London, UK \\ ${ }^{13}$ UCL Institute of Ophthalmology, London, UK
}

Correction notice This article has been updated since it was published online. The fifth author's surname has been corrected.

Acknowledgements Additional GLAUrious principal investigators include Noa Geffen, MD, Zohar Bracha, MD, Maya Kalev-landoy, MD, Assaf Kratz, MD, Gabriel Greifner, MD, Shiri Shulman, MD (Israel); Merav Dvali, MD, Vakhtang Javrishvili, MD, Anna Dvalishvili, MD (Georgia). The authors acknowledge Helen Buck, PhD, for writing assistance, funded by Belkin Limited.

Contributors All authors were involved in the development of the study protocol, all acted as principal investigators on the study except TA, SJF, RL, TS, KS and YS. All of the authors have been closely involved and offered expertise and advice during the development of the Eagle device.

Funding This work was supported by an EU Horizon 2020 Grant (grant 720274) and by the Study Sponsor Belkin Laser.
Competing interests This work was partially supported by an EU Horizon 2020 Grant (grant 720274) and was supported by the Study Sponsor Belkin Laser.

Patient consent for publication Not required.

Provenance and peer review Not commissioned; externally peer reviewed.

Data availability statement No data are available. No data are available as this is a protocol submission.

Open access This is an open access article distributed in accordance with the Creative Commons Attribution Non Commercial (CC BY-NC 4.0) license, which permits others to distribute, remix, adapt, build upon this work non-commercially, and license their derivative works on different terms, provided the original work is properly cited, appropriate credit is given, any changes made indicated, and the use is non-commercial. See: http://creativecommons.org/licenses/by-nc/4.0/.

\section{ORCID iDs}

Nathan Congdon http://orcid.org/0000-0001-9866-3416

Augusto Azuara-Blanco http://orcid.org/0000-0002-4805-9322

Michele lester http://orcid.org/0000-0002-0524-2637

Carlo Alberto Cutolo http://orcid.org/0000-0002-2433-0704

\section{REFERENCES}

1 Tham Y-C, Li X, Wong TY. Global prevalence of glaucoma and projections of glaucoma burden through 2040: a systematic review and meta-analysis. Ophthalmology 2014;121:2081-90.

2 Burton MJ, Ramke J, Marques AP, et al. The Lancet global health Commission on global eye health: vision beyond 2020. Lancet Glob Health 2021;9:e489-551.

3 Feehan M, Munger MA, Cooper DK, et al. Adherence to glaucoma medications over 12 months in two US community pharmacy chains. J Clin Med 2016;5:79.

4 James Lind Alliance. Glaucoma top 10 | James Lind alliance, 2021. Available: https:// www.jla.nihr.ac.uk/priority-setting-partnerships/sight-loss-and-vision/top-10priorities/glaucoma-top-10.htm

5 Gazzard G, Konstantakopoulou E, Garway-Heath D, et al. Selective laser trabeculoplasty versus eye drops for first-line treatment of ocular hypertension and glaucoma (LiGHT): a multicentre randomised controlled trial. The Lancet 2019;393:1505-16.

6 Garg A, Vickerstaff V, Nathwani N, et al. Efficacy of repeat selective laser trabeculoplasty in Medication-Naive open-angle glaucoma and ocular hypertension during the light trial. Ophthalmology 2020;127:467-76.

7 Investigators A. The advanced glaucoma intervention study (AGIS): 4. Comparison of treatment outcomes within race. seven-year results. Ophthalmology 1998; 105:1146-64.

8 Geffen N, Ofir S, Belkin A, et al. Transscleral selective laser trabeculoplasty without a gonioscopy lens. J Glaucoma 2017;26:201-7.

9 Goldenfeld M. Automated direct selective laser trabeculoplasty -First in human prospective clinical study, 2021. Available: https://ascrs.confex.com/ascrs/21am/ ascrspaper/papers/viewonly.cgi? password $=901880$ \&username $=74383$

10 Stewart W, Stewart J, Nelson L. Glaucoma clinical trial design: a review of the literature. Perspect Clin Res 2014;5:108-14.

11 Burr JM, Botello-Pinzon P, Takwoingi Y, et al. Surveillance for ocular hypertension: an evidence synthesis and economic evaluation. Health Technol Assess 2012;16:1-127.

12 Cook JA, Botello AP, Elders A, et al. Systematic review of the agreement of tonometers with Goldmann applanation tonometry. Ophthalmology 2012;119:1552-7.

13 Eyawo $\mathrm{O}$, Lee C-W, Rachlis B, et al. Reporting of noninferiority and equivalence randomized trials for major prostaglandins: a systematic survey of the ophthalmology literature. Trials 2008:9:69.

14 Latina MA, Sibayan SA, Shin DH, et al. Q-switched 532-nm Nd:YAG laser trabeculoplasty (selective laser trabeculoplasty). Ophthalmology 1998;105:2082-90.

15 Woo DM, Healey PR, Graham SL. Intraocular pressure-lowering medications and longterm outcomes of selective laser trabeculoplasty: glaucoma laser and medical therapy. Clin Experiment Ophthalmol 2015;43:320-7.

16 El Mallah MK, Walsh MM, Stinnett SS, et al. Selective laser trabeculoplasty reduces mean IOP and IOP variation in normal tension glaucoma patients. Clin Ophthalmol 2010;4:889-93.

17 Goldenfeld M, Geyer O, Segev E, et al. Selective laser trabeculoplasty in uncontrolled pseudoexfoliation glaucoma. Ophthalmic Surg Lasers Imaging 2011;42:390-3.

18 Wong MOM, Lee JWY, Choy BNK, et al. Systematic review and meta-analysis on the efficacy of selective laser trabeculoplasty in open-angle glaucoma. Surv Ophthalmol 2015;60:36-50.

19 Martinez-de-la-Casa JM, Garcia-Feijoo J, Castillo A, et al. Selective vs argon laser trabeculoplasty: hypotensive efficacy, anterior chamber inflammation, and postoperative pain. Eye 2004;18:498-502.

20 Shi J-M, Jia S-B. Selective laser trabeculoplasty. Int J Ophthalmol 2012;5:742-9.

21 Kramer TR, Noecker RJ. Comparison of the morphologic changes after selective laser trabeculoplasty and argon laser trabeculoplasty in human eye bank eyes. Ophthalmology 2001;108:773-9. 